\title{
Utilisation des lasers femtosecondes pour le marquage du verre, dans le cadre de la lutte contre la contrefaçon
}

L'utilisation d'impulsions ultracourtes (de l'ordre de quelques dizaines de femtosecondes, 1 femtoseconde = $10^{-15}$ seconde) a ouvert de nombreuses perspectives à la fois industrielles et scientifiques, en particulier pour la micro-/nanostructuration de surface, ou la modification structurale dans le volume pour les matériaux transparents.

Hervé SODER

Impulsion

contact@impulsion-sas.com

Éric AUDOUARD

Impulsion - Université de Lyon - Université de Saint-Étienne/Laboratoire Hubert Curien

\section{Importance industrielle du marquage et spécificité des procédés femtosecondes}

Le marquage laser a été très tôt considéré comme un domaine d'intérêt pour les technologies femtosecondes. II est possible d'atteindre, en mode femtoseconde, le seuil d'ablation laser de tous types de matériaux : métaux spéciaux et céramiques, matériaux organiques ou polymères utilisés par exemple en milieu médical, céramiques piézoélectriques, silicium pour la microélectronique, métaux pour la micromécanique, mais aussi dans le domaine des matériaux transparents. La modification structurale ou l'ablation sont alors très précises (de l'ordre du micromètre et même inférieure), sans altération de la zone non traitée par le laser. II est possible de graver la surface des verres en contrôlant la profondeur à quelques dizaines de nanomètres près, mais aussi de modifier dans la masse l'indice desmatériaux transparents, et de réaliser ainsi des guides d'onde, ou plus largement de fonctionnaliser les matériaux. La nature de l'interaction laser matière en mode ultra- court est fondamentalement différente d'une interaction avec des impulsions plus longues (nanosecondes).

Cette différence fondamentale fait aujourd'hui l'objet de nombreux travaux de recherche et conduit à l'émergence de nouvelles technologies innovantes dans le domaine des procédés lasers.

\section{Marquage dans le domaine des matériaux transparents}

Les technologies lasers ont un intérêt particulier dans le domaine des matériaux transparents. Pour ce type de matériaux, les lasers utilisés habituellement, de durée d'impulsion nanoseconde, ont le plus souvent des longueurs d'onde dans l'ultraviolet. Il est alors possible, par focalisation, de graver dans la masse, en 3D, toutes sortes d'images ou d'objets. Un photon ultraviolet, beaucoup plus énergétique qu'un photon infrarouge,

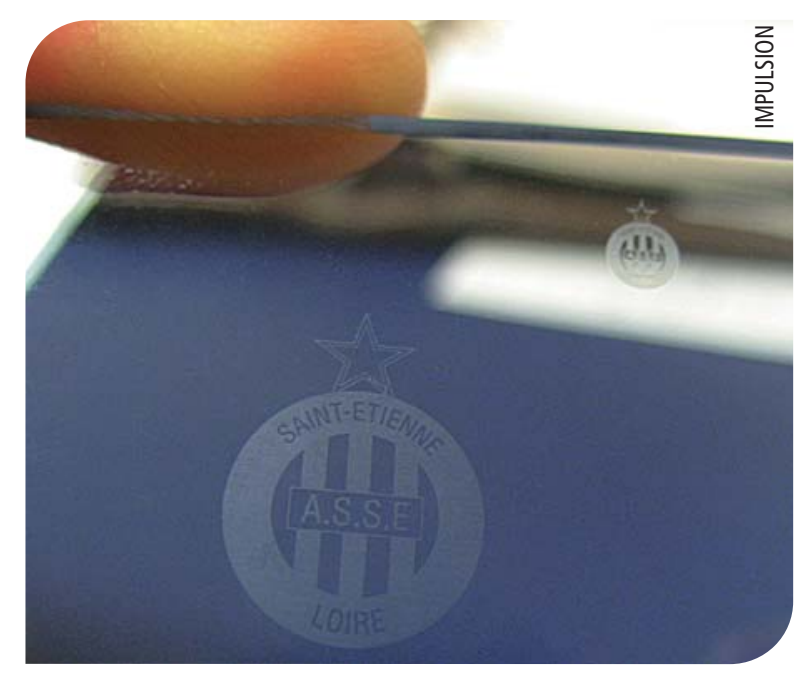

Figure 1. Exemple de marquage sur verre par impulsion ultra brève, illustrant la maîtrise de l'échelle. peut rompre la cohésion d'un solide sans passer par les étapes caractéristiques des mécanismes thermiques. Le laser réalise ainsi localement des microcassures qui rendent visible à l'œil le parcours du faisceau. Un bon contrôle des paramètres du procédé peut également conduire à une simple modification interne du matériau, conduisant par exemple à la modification locale de l'indice du matériau. On parle alors de photoinscription. Dans le cas de l'utilisation d'impulsions ultrabrèves pour la photoinscription, contrairement aux techniques de microgravure, le but recherché n'est pas de vaporiser la matière mais de s'arrêter à la fusion locale du verre. Ensuite, la resolidification s'accompagne d'une densification locale qui entraîne une augmentation de l'indice dans la zone irradiée. L'effet, encore mal compris, repose sur une excitation multiphotonique ultrarapide qui semble fournir suffisamment d'énergie aux électrons puis, par couplage, au réseau, pour atteindre le point de fusion. Contrairement aux procédés de variation d'indice photoinduite dans les verres par laser UV, technique qui a entre autres révolutionné les dispositifs de communication optique dans les années 1990, avec en particulier toute une technologie développée autour de l'inscription de réseaux de Bragg, la modification d'indice photoinduite par laser femtoseconde à $800 \mathrm{~nm}$ n'est pas limitée aux surfaces car les matériaux n'absorbent pas à cette longueur d'onde. Ainsi, des dispositifs enterrés, et donc 
peu sensibles aux perturbations, ou bien des composants 3D sont accessibles. De plus, la variation d'indice induite est très locale du fait même de son origine physique (figure 1). Les variations d'indice maximales mesurées et atteignent par exemple $\Delta \mathrm{n}=3 \times 10^{-3}$ dans des verres borosilicates.

Bien sûr, les impulsions ultrabrèves peuvent également être utilisées pour du marquage de surface, y compris par enlèvement de matière. L'intérêt d'une telle technique est alors de minimiser les microcraquelures souvent observées pour ce type de réalisations.

\section{Spécificités de l'ultrabref pour le domaine du marquage}

Le domaine du marquage est un domaine très actif des applications femtosecondes. C'est un secteur industriel en fort développement, pour les besoins d'identification et de traçabilité de la production industrielle et de la lutte contre la contrefaçon.

\section{Marquage de petite taille}

Les technologies femtosecondes trouvent dans ce secteur un domaine naturel d'application par leur capacité à modifier les matériaux dans la masse et à atteindre des précisions importantes. Par exemple, la photoinscription par laser femtoseconde de codes à deux dimensions dans la masse de matériau transparent, appelée Datamatrix, est un domaine où sont présentes plusieurs sociétés commerciales. L'intérêt des impulsions ultrabrèves est de permettre un marquage très petit, pouvant aller jusqu'à une taille submicronique. On reste donc dans le cadre de marquage par modification permanente et visible du matériau mais à une taille invisible à I'œil, ce qui permet soit une dissimulation du marquage, soit une densification de l'information inscrite dans le code marqué. Bien sûr, il convient également d'adapter en parallèle les techniques de relecture de l'information, ce qui demande un travail d'optique/ vision spécifique.

\section{Contrôle jusqu’à la modification de l'indice : marquage invisible}

Les marquages par microcassures, qui permettent donc une inscription par laser nanoseconde dans la masse des matériaux transparents, sont obtenus par concentration ponctuelle d'énergie. Grâce à une technique de déplacement du faisceau point par point ou par tracé linéaire, n'importe quelle forme peut être photoinscrite. Cette technique est devenue populaire par la réalisation d'objets décoratifs ; elle peut bien sûr être également utilisée pour l'identification de produits manufacturés. Dans l'exemple de la figure 2, la figure, imprimée par le même type de déplacement de faisceau, et toujours à cause d'une concentration d'énergie ponctuelle, n'est pourtant pas visible sans une technique optique adaptée. L'impulsion ultrabrève modifie alors l'indice de réfraction du matériau. L'origine de la variation d'indice photoinduite dans les milieux diélectriques par des impulsions femtosecondes reste encore incomplètement $\gg$

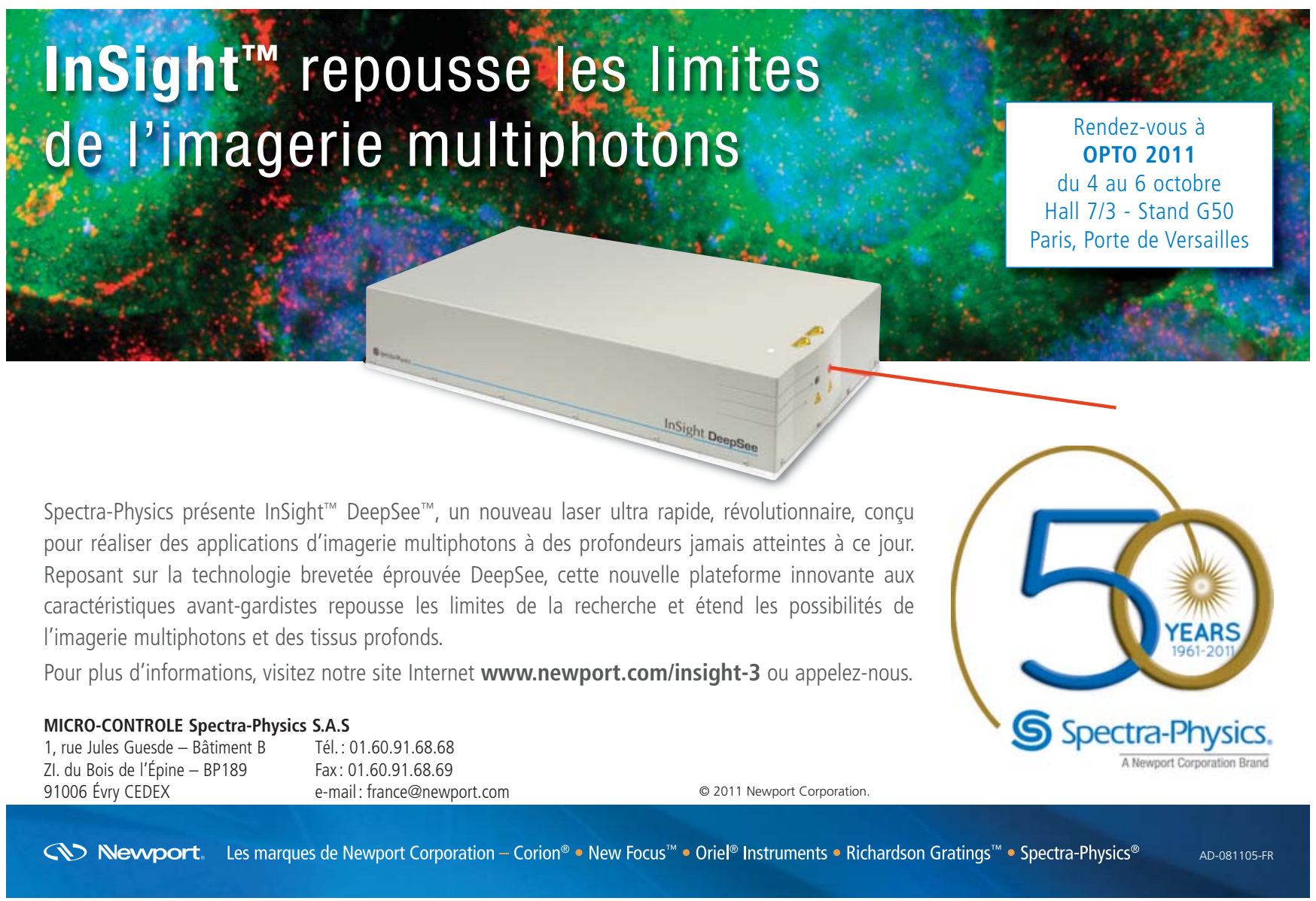




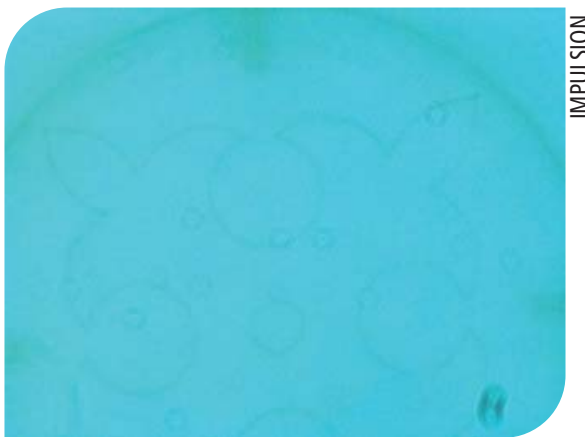

Figure 2. Exemple de marquage par modification d'indice : mire de $2 \mathrm{~mm}$ de diamètre vue au microscope à contraste de phase. On peut distinguer un motif au centre, qui n'apparaît pas au microscope à rétro-éclairage.

expliquée : formation de défauts, densification locale, contraintes... Des analyses expérimentales ont été faites en régime permanent (longtemps après l'exposition) ou résolues en temps par microscopie à contraste de phase. En particulier, il y a coexistence de zones d'augmentation (zone sombre) et de diminution d'indice (zone claire) dans la zone d'interaction.

Pour parvenir au contrôle des procédés, une compréhension des mécanismes physiques dans les diélectriques est nécessaire. Cela passe par une modélisation de la variation d'indice. Ce travail très conséquent couvre d'abord le pro- blème de la répartition d'énergie dans la zone irradiée. Des effets non linéaires tels que la filamentation ou la défocalisation par le plasma d'électrons doivent être pris en compte, sans oublier les aberrations géométriques (aberration sphérique). Les premiers résultats des codes numériques sont comparés aux observations de variation d'indice induite. La description des phénomènes mécaniques, thermodynamiques et électroniques pouvant induire une variation $d$ 'indice doit aussi être prise en compte.

\section{Développement/recherche : mise en forme temporelle - contrôle de la variation d'indice}

Des outils de mise en forme temporelle d'impulsion femtoseconde autoadaptative ont également été mis au point, et trouvent des applications particulièrement intéressantes dans le cas des matériaux transparents [1]. La mise en forme temporelle est réalisée en intégrant une valve optique adressée électriquement (figure 3). L'impulsion ainsi mise en forme temporellement est focalisée sur un échantillon pour étudier un procédé donné. Nous nous intéressons ici à des matériaux diélectriques, dans le but $d^{\prime}$ 'optimiser la variation d'indice induite. Le procédé est analysé en temps réel par un microscope à contraste de phase couplé à une caméra, puis évalué par une unité de traitement qui compare le profil d'indice photoinduit avec un profil cible, prédéfini par l'utilisateur. Une rétroaction sur le profil de phase spectrale est établie et la convergence vers le profil cible est assurée par un algorithme itératif évolutionnaire.

On dispose ainsi d'un outil précieux pour l'exploration de l'interaction lasermatériau et pour l'optimisation des procédés avec rétroaction sur le résultat même du procédé. La mise en forme temporelle auto adaptative a alors été utilisée pour optimiser la variation d'indice induite dans des matériaux transparents. Certains résultats très particuliers ont ainsi pu être mis en évidence : le BK7 est normalement le siège d'une variation d'indice photoinduite négative. Pourtant, en appliquant la séquence excitatrice adéquate, il est possible d'induire dans du BK7 le même type de profil d'indice que dans la silice (à savoir positive !). Il est donc possible d'inscrire une structure guidante dans un matériau qui à l'origine ne présente aucune prédisposition à la photoinscription. De même, l'aptitude à réaliser une photoinscription dans la masse en trois dimensions ouvre des perspectives intéressantes.

Analyse du résultat Commande de la mise en forme temporelle par algorithme évolutionnaire
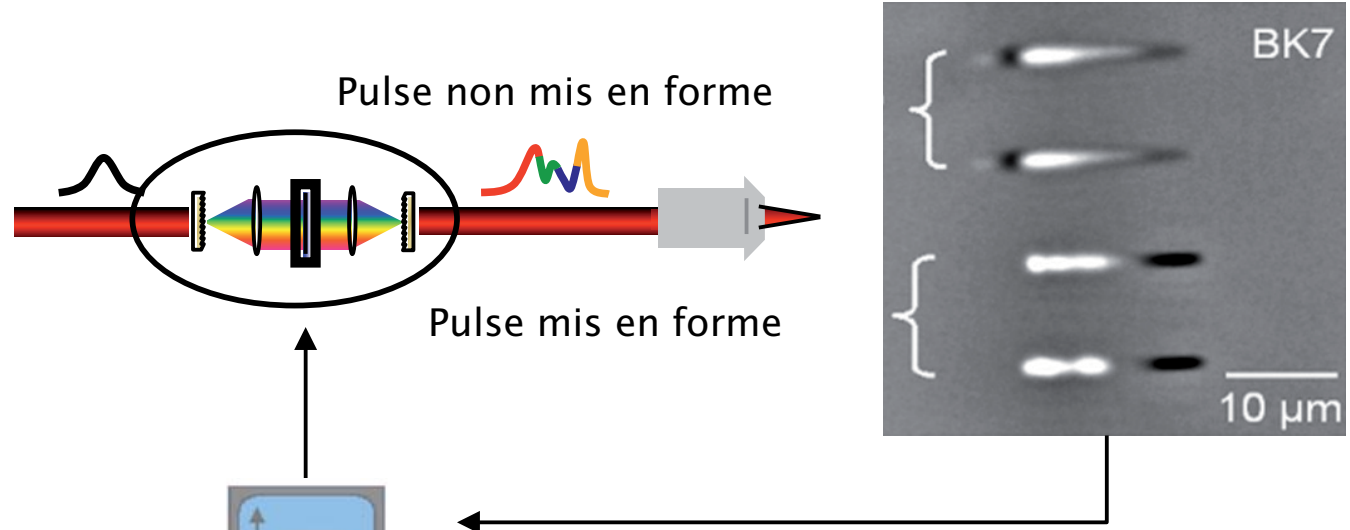

Pulse mis en forme

Comparaison avec l'optimum

Figure 3. Principe de la mise en forme temporelle autoadaptative pour le contrôle de la variation d'indice dans les milieux transparents [1]. 


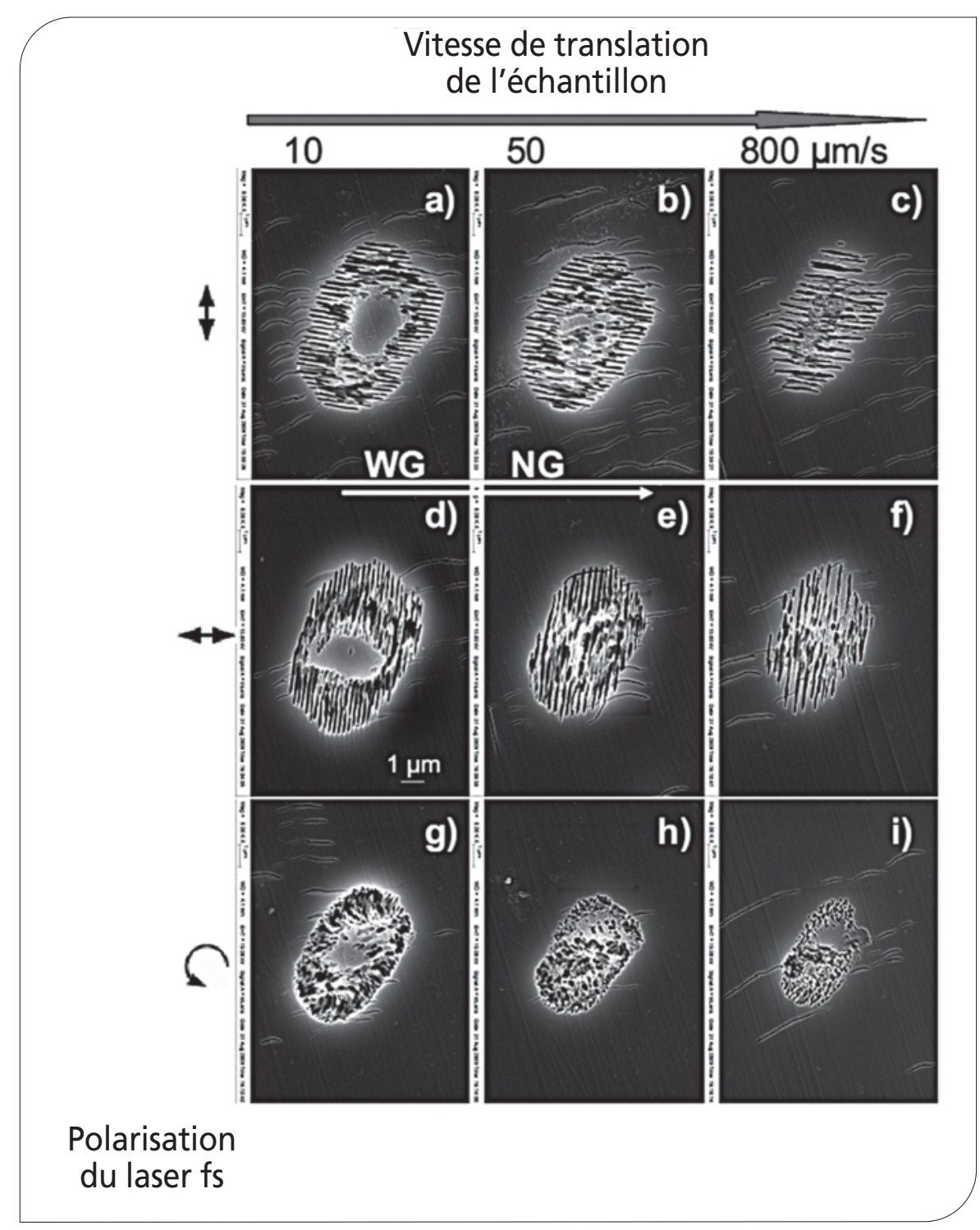

Figure 4. Exemple de nanostructures de type « ripples » obtenues dans la masse de matériaux transparents [3].

\section{Nanostructures dans la masse}

L'effort pour aller vers des tailles toujours plus petites est soutenu par la mise au point des outils de contrôle spatial que nous avons évoqués ci-dessus. Une résolution allant jusqu'à la centaine de nanomètres est tout à fait accessible dans de nombreux centres laser. L'utilisation d'objectifs spéciaux et de platines de déplacement précises permet d'aller jusqu'aux limites de dispositif de micro/nanostructuration, ce qui reste cependant crédible pour des applications de nature industrielle. On peut citer dans ce sens la constitution du consortium européen FemtoPrint [2]. Un effet spécifique des impulsions ultrabrèves peut en effet conduire à des applications très innovantes. Il s'agit de la formation de microstructures périodiques, appelées "ripples», dans les cratères d'ablation ou en surface des matériaux. Ces ondulations présentent un intérêt particulier car on les retrouve sous différentes formes dans de très nombreux résultats d'expériences d'ablation en mode femtoseconde. Ces nanostructures «pilotables» par la polarisation s'obtiennent sur presque tous les métaux, leur morphologie dépend surtout de la longueur d'onde incidente. Elles s'obtiennent aussi pour les matériaux diélectriques, en surface et dans la masse des matériaux. Ces structures peuvent être pilotées par la polarisation, comme le montrent par exemple les travaux de R. Stoian et al. [3] illustrés dans la figure 4. La compréhension de la formation de ces structures est un sujet d'étude très actif, la question qui se pose est de savoir comment une caractéristique du champ électromagnétique (la polarisation) qui n'existe que dans quelques dizaines de femtosecondes peut se retrouver « imprimée » dans la matière qui demande plusieurs picosecondes pour se mettre en mouvement.

\section{Conclusion}

Nous venons de montrer que les technologies femtosecondes peuvent apporter des solutions originales et innovantes dans le domaine du marquage, ce qui représente une contribution importante pour la lutte contre la contrefaçon par identification et traçabilité des produits manufacturés. Bien évidemment, deux écueils doivent encore être dépassés : le coût des sources femtosecondes et les techniques de relecture. Une solution au premier point peut résider dans l'utilisation de sources à hautes cadences puisque les "process» envisagés sont peu gourmands en énergie. La rapidité d'exécution des marquages est par ailleurs une exigence industrielle. Les problèmes de relecture peuvent être associés au marquage d'éléments de petite taille ; la mise au point de méthodes spécifiques doit donc être envisagée. Le domaine du marquage laser, en forte expansion actuellement, peut doncs'appuyer dans les années à venir sur un fort potentiel de renouvellement technologique.

\section{Références}

[1] R. Stoian, A. Mermillot-Blondin, S.W. Winkler, A. Rosenfeld, I.V. Hertel, M. Spyridaki, E. Koudoumas, P. Tzanetakis, C. Fotakis, I.M. Burkanov, N.M. Bulgakova. Temporal pulse manipulation and consequences for ultrafast laser processing of materials. Optical Engineering 2005; 44 (5): 051106.

[2] http://www.femtoprint.eu/

[3] K. Mishchik, G. Cheng, G. Huo, M. Burakov, C. Mauclair, A. Mermillod-Blondin, A. Rosenfeld, Y. Ouerdane, A. Boukenter, 0. Parriaux, and R. Stoian. Nanosize structural modifications with polarization functions in ultrafast laser irradiated bulk fused silica. Optics Express 2010; 18(24): 24809-24824. 